\title{
Meetings / Réunions
}

\section{DECEMBER 2000}

- Annual conference, International Society of Tropical Foresters, 4 December.

Washington, DC. Contact: Dr. Warren T. Doolittle, President, ISTF, 5400 Grosvenor Lane, Bethesda, MD 20814. Fax: 1-301897-3690; e-mail: istf@igc.apc.org; Web site: www.cof.orst.edu/org/istf

\section{- Western Forestry Conference, 4-5 December.}

Coeur d'Alene, Idaho, U.S.A. Contact: Richard Zabel, Western Forestry and Conservation Association, 4033 SW Canyon Rd., Portland, Oregon 97221: Tel: 503-2264562; Fax: 503-226-2515; e-mail: richard@ westernforestry.org

- Integrated management of neotropical rain forests by industries and communities, 4-8 December.

Belém, Brazil. Contact: Dr. Natalino Silva, Fax: 55-91-226-9845; e-mail: natalino@ epatu.embrapa.br

- 5th Pacific Rim bio-based composites symposium, 10-13 December. Canberra, ACT, Australia. Contact: Dept. of Forestry, Australian National University, Canberra ACT 0200, Australia. E-mail: bio.symposium@anu.edu.au

\section{1}

- Resources, trade and market structure for bamboo and rattan, Fall, 2001. Beijing, China. Contact: Mr. Lou Yiping, International Network for Bamboo and Rattan (INBAR), Anyuan Building No. 10, Anhui Beilj, Asian Games Village, Beijing, China; e-mail: yplou@inbar.org.cn; Web site: http://www.inbar.org.cn

- Diseases and insects in forest nurseries (IUFRO), date TBA.

Kerala, India. Contact: Stephen Fraedrich, USDA Forest Service, 320 Green Street, Athens, GA 30602., USA. Fax: 1-706-5594287; E-mail: fraedrich_stephen/srs_ athens@fs.fed.us

\section{JANUARY 2001}

Landscape legacies: managing landscapes in the context of human history and ecosystem change, 8-10 January.

Durham, North Carolina. Contact: Susan Fox; Tel: 919-513-3331; e-mail: sfox@ unity.ncsu.edu
- Forest inventory and GIS: Real challenges, practical solutions, 9-10 January.

Portland, Oregon, USA. Contact: Richard Zabel, Western Forestry and Conservation Association, 4033 SW Canyon Road, Portland, Oregon 97221, USA. Fax: 503-226-2515; e-mail: richard@westernforestry.org

- New knowledge and solutions for forest management, 29-30 January. Timmins, Ontario Canada. Contact: Trevor Griffin; Fax: 705-272-7811; e-mail: workshop@lamf.net; Web site: www.lamf.net

\section{FEBRUARY 2001}

- Global change and sustainable development in Southeast Asia, 17-19 February.

Chiangmai, Thailand. Contact: Dr. Louis Lebel, Chiangmai University, Chiangmai, Thailand 5000. Tel/Fax: 66-53-263-215; e-mail: llebel@cmnet.co.th. Web Site: www. icsea.or.id/sarcs2001/

GIS 2001 - branching out: Spatial technology goes mainstream, 19-22 February.

Vancouver, B.C., Canada. Contact: Canadian Institute of Geomatics: Fax 613-224-9577; e-mail: executive.director@cig-acsg.ca

- Politics and realities of wildlife conservation at the start of the 21st century, 22-24 February.

Sacramento, California USA. Contact: Barry Garrison, Tel: 916-653-1738; e-mail: bagarris@ dfg.ca.gov

- Taking Nature Seriously: Citizens, Science, and Environment, 25-27 February.

Eugene, Oregon: Contact: E-mail: tns@darkwing.uoregon. edu

\section{MARCH 2001}

Enhanced forest management conference-fertilization and economics, 1-2 March.

Edmonton, Alberta, Canada. Contact: Tel: 780-1858; e-mail: clear@compusmart.ab.ca; Website: www.compusmart.ab.ca/clear/efm.html

- Riparian habitat and floodplains conference, 12-15 March.

Sacramento, California USA. Contact: Diana Craig, USDA Forest Service: Tel: 707-5628930; e-mail: dcraig01@fs.fed.us
- 4th South and East Asian countries Non-Timber Forest Products Network (SEANN) workshop, 18-21 March.

Manila, Philippines. Contact: Dr. Ramon A. Razal, College of Forestry and Natural Resources, UP Los Banos, College, Laguna, Philippines; Fax: 63-49-536-3340; e-mail: trees@laguna.net

- Economic sustainability of smallscale forestry, 20-26 March.

Joensuu, Finland. Contact: Dr. Anssi Niskanen, European Forest Institute, Tonkatu 34, 80100 Joensuu, Finland. Fax: 358-13-124-393; e-mail: anssi_niskanen@efi.fi

- Building sustainable communities: Regional and watershed approaches, 21-23 March.

North Adams, Massachusetts, USA. Contact: Mervin Stephens Berkshire Institute at Greylock, Box 214, Adams, MA 01220 , USA. Fax: 919-682-2349; e-mail: stevens@ duke.edu; Website: www.lib.duke.edu/ forest/

- Global initiatives and public policies: First international conference on private forestry in the 21st Century, 25-27 March.

Atlanta Georgia. Contact: Larry Teeter, School of Forestry and Wildlife Sciences, Auburn University, $201 \mathrm{M}$. White Smith Hall, Auburn, AL 36849-5418; Tel: 334 844-1045; Fax: 334-844-1084

- Wilderness Britain: Social and environmental perspectives on recreation and conservation, 26-28 March. Leeds, West Yorkshire, UK. Contact: Dr. Steve Carver, University of Leeds. Fax: 44-(0)-113233-3308; e-mail: steve@geog.leeds.ac.uk

- Making environmental history relevant in the 21st century, 28 March-1 April.

Durham, North Carolina, U.S.A. Contact: Web site, www.lib.duke.edu/forest/

\section{APRIL 2001}

- The Australasian Conference on tree and nut crops, 23-20 April.

Perth, Western Australia. Contact: Web: www.aoi.com.au/acotanc/acotanc2001.htm

16th Commonwealth forestry conference, 18-25 April. 
Fremantle, Perth, Western Australia. Contact: Commonwealth Forestry Association, Oxford Forestry Institute, South Parks Rd., Oxford OX1 3RB, UI; Fax: 44-1865-275074; e-mail: cfa@plants.ox.ac.uk

\section{MAY 2001}

- Tree structure and mechanics: how trees hold together and fall apart, 6-9 May.

Savannah, Georgia. Contact: Dr. Kim Coder, University of Georgia. E-mail: kcoder@ arches.uga.edu

- Canadian Water Resource Association - B.C. Branch Conference, Changing water environments: research and practice, 8-11 May.

Whistler, B.C. Contact: Paul H. Whitfield: Tel: 604-664-9238; Fax: 604-664-9125; e-mail: paul.whitfield@ec.gc.ca; Web site: www.cwra.org

- North American forest insect work conference, 14-18 May.

Edmonton, Alberta, Canada. Contact: Jan Volney, Canadian Forest Service. Tel: 780435-7329; e-mail: jvolney@nrcan.gc.ca

- 6th international conference on woodfiber-plastic composites, 15-16 May.

Madison, Wisconsin, USA. Contact: Julie Lang, Forest Products Society, 2801 Marshall Court, Madison, WI 53705-2295: Fax: 608231-2152; e-mail: conferences@forestprod.org

- Ecosystem management in boreal forest landscapes, 27-30 May 2001.

North Karelia, Finland. Contact: Brita Pjari: Fax: 358-0-13-124-397; e-mail: brita.pajari@efi.fi; Website: www.joensuu.fi/forestconference/

\section{JUNE 2001}

- FAO/ECE/ILO workshop on new developments of wood harvesting with cable systems, June (dates to be announced).

Austria (city to be announced). Contact: R. Heinrich, Forest Products Division, FAO, Viale delle Terme di Caracalla, 00100 Rome, Italy; Fax: 39-06-5705 5137; e-mail: forestharvesting@FAO.org

- 8th International conference on biotechnology in the pulp and paper industry, 4-8 June.

Helsinki, Finland. Contact: Prof. Liisa Viikari, VTT Biotechnology, Box 1500, FIN-02044, Finland. Fax: 358-9-455-1203.
Elmia Wood 2001 - international forestry trade fair, 6-9 June.

Jonkoping, Sweden. Contact; Torbjorn Johnsen; Fax: 46-36-16-46-92; e-mail: wood@elmia.se; Website: www.wood.elmia.se

- International conference on ex situ and in situ conservation of commercial tropical trees, 11-13 June.

Yogyakarta, Indonesia. Contact: Ms Soetitah S. Soedojo; Fax: 62-274-902 220; e-mail: itto-gmu@yogya.wasantara.net.id

- Foliage shoot and stem diseases of trees, 16-24 June.

Korkeakoski, Finland. Contact: Antti Uotila, Fax: 358-31-335-5555; e-mail: auotila@ hyytiala.helsinki.fi

- Avalanche control on the base of hazard mapping in avalanche areas, 18-21 June.

Rauris, Austria. Contact: Gernot Fiebiger or Erich Scheuringer: Fax: 43-662-870215; e-mail: fiebigerf@cunet.at

\section{- Forest Products Society Annual Meeting, 24-27 June.}

Baltimore, Maryland, U.S.A. Contact: Dr. Richard Vlosky, School of forestry, Wildlife, and Fisheries, Louisiana State University, Baton Rouge, LA 70803, U.S.A.; Tel: 225-388-4527; Fax: 225-388-4251; e-mail: vlosky@ Isu.edu

\section{JULY 2001}

- Travelling workshop on linking the complexity of forest canopies to ecosystems and landscape function, 11-19 July.

Portland and Corvallis, Oregon. Contact: Michael G. Ryan, Rocky Mountain Research Station, 240 West Prospect RD, Fort Collins, CO 80526-2098. Fax: 970-498-1027; e-mail: mryan@lamar.colostate.edu

- Tree biotechnology: the next millennium, 22-27 July.

Stevenson, Washington, U.S.A. Contact: Dr. Stephen Strauss, Department of forest Science, Oregon State University, Corvallis, Oregon 97331-7501. Fax: 541-737-1393; e-mail: strauss@fsl.orst.edu; Web site: www.cof.orst.edu/cof/extended/conferen/treebio//

\section{AUGUST 2001}

- The western forest genetics Association Conference, 30 July-2 August. Davis, California. Contact: Dr. David Neale, USDA Forest Service, Tel: 530-754-8431; e-mail:dneale@dendrome.ucdavis.edu
- The role of bamboo in disaster avoidance, 30 July-3 August.

Quito, Ecuador. Contact: Mr. Lou Yiping, INBAR, Branch Box 155, P.O. Box 9799, Beijing 100101, China. Fax: 0086-10-64956962; e-mail: yplou@inbar.org.cn; Web: www.inbar. org.cn

- Forest modelling for ecosystem management, forest certification, and sustainable development, 12-18 August. Vancouver, B.C. Canada: Contact: Dr. Valerie LeMay; e-mail: forestmd@interchange.ubc.ca; Website: www.forestry.ubc.ca/forestmodel

\section{SEPTEMBER 2001}

$\checkmark$ 49th annual meeting of the Western International Forest Disease Work Conference, September (dates, TBA). Monterray area, California. Contact: Susan Frankel, USDA Forest Service, 1323 Club Drive, Allejo, CA 94592. Fax: 707-562-9054; E-mail: sfrankel/r5@fs.fed.us

- Forest recreation, landscape and nature conservation (IUFRO), September, (dates TBA).

Southeastern USA (TBA). Contact: John Burde, School of Forestry, Southern Illinois University, Carbondale, IL 62901, USA Fax: 618-453-7475; e-mail: jburde@ siu.edu.

- 82nd annual conference of the Canadian Woodlands Forum, 5-7 September.

Quebec City, QC, Canada. Contact: Guillaume Gignac, Executive Director, Canadian Woodlands Forum. Tel: 514-392-6946; E-mail: ggignac@cwfcof.org; Web Site: www.cwfcof.org

5th international flora malesiana Symposium, 9-14 September.

Sydney, Australia. Contact: Dr. Barry Conn, Royal Botanic Gardens Sydney, Mrs. Macquaries Road, Sydney NSW 2000, Australia.E-mail: fmv@rbgsyd.gov.au; Web site: http://plantnet.rbgsyd.gov.au/fm/fm.html

\section{- Dynamics of forest insect popula-} tions, 10-14 September.

Aberdeen, Scotland. Contact: Andrew Liebhold, USDA Forest Service, 180 Canfield Street, Morgantown, West Virginia 26505 USA: Fax: 304-285-1505; e-mail: sandy@ gypsy.fsl.wvu.edu

- Dynamics of forest insect populations, 12-14 September.

Aberdeen, Scotland, U.K. Contact: Dr. Andrew Liebhold, Forestry Sciences 
Laboratory, 180 Canfield St., Morgntown, West Virginia 26505. Fax: 304-285-1505; e-mail: sandy@gypsy.fsl.wvnet.edu;

- Root and butt rots (IUFRO), 17-21 September.

Quebec City, Quebec, Canada. Contact: Gaston Laflamme, Canadian Forest Service, 1055, rue du P.E.P.S./PO Box 3800, SainteFoy, Quebec G1V 4C7, Canada; Fax: 418648-5849; e-mail: glaflamme@cfl.forestry.ca

- Tree rings and people-an international conference on the future of dendrochronology, 22-26 September. Davos, Switzerland. Contact: Paolo Cherubini: Fax: 41-1-7392215; e-mail: paolo.cherubini@ wsl.ch

- Uneven-aged silviculture tradition and practices in central Europe, 24-30 September.

Zurich, Switzerland. Contact: Andreas Zingg, Swiss Federal Research Institute, Zurcherstr. 111, CH-8903 Birmensdorf, Switzerland. Fax: 41-1-739-22-15; e-mail: andreas. zingg@wsl.ch; Web site: www.wsl.ch/ forest/waldman/events/

\section{OCTOBER 2001}

- Improvement and culture of eucalypts, October (dates to be specified).

Valdivia, Chile. Contact: Dr. Roberto Ipinza, Universidad Austral de Chile, P.O. Box 1241, Valdivia, Chile. Fax: 56-63-224-677; e-mail: ripinza@valdivia.uca.uach.cl

- Continuous cover forests - assessment, analysis, scenarios, 10-12 October.

Gottingen, Germany. Contact: Mrs. Stachowiak, e-mail: elojews@uni-forst.gwdg.de.

- National symposium on "Old-growth Forests in Canada - A Science Perspective", 15-19 October 2001.

Sault Ste. Marie, Ontario, Canada. Contact: Dr. Bruce Pendrel, Canadian Forest Service, P.O. Box 4000, Fredericton, N.B., Canada: Tel: 506-452-2440; Fax: 506-452-3525; e-mail; oldgrowth@nrcan.gc.ca

\section{NOVEMBER 2001}

- VII international bamboo workshop and congress, November (exact dates TBA).

India (city TBA). Contact: Dr. I.V. Ramanuja Rao, Branch Box 155, P.O. Box 9799, Beijing 100 101, China. Fax: 86-10-6495-6962/83; e-mail: rrao@inbar.org.cn.
DECEMBER 2001

- International Society of Tropical Foresters, annual conference, 3 December.

Washington, D.C. Contact: Dr. Warren T. Doolittle, President, ISTF, 5400 Grosvenor Lane, Bethesda, MD 20814, U.S.A. Fax: 301-897-3690; e-mail: istfiusf@igc.apc.org; Web site: www.cof.orst.edu/org/istf

\section{2}

- III International rattan congress, 2002 (dates TBA).

Phillipines or Malaysia (tba). Contact: Dr. I.V. Ramanuja Rao, Branch Box 155, P.O. Box 9799, Beijing 100 101, China. Fax: 86-106495-6962/83; e-mail: rrao@inbar.org.cn.

\section{FEBRUARY 2002}

- Sustaining livelihoods and biodiversity in the new millennium, 12-15 February.

Phnom Penh, Cambodia. Contact: Devin Bartley, FAO, Via delle Terme di Caracalla, Rome 00100, Italy. E-mail: devin.bartley@ fao.org

\section{AUGUST 2002}

- Methodology of forest insect and disease survey in Central Europe, August, 2002.

Krakow, Poland. Contact: Michael McManus, USDA Forest Service, Hamden, Conn., U.S.A.; Fax: 1-203-230-4315; e-mail: mmcmanus/ne_ha@fs.fed.us
- 26th international horticultural Congress, 11-17 August.

Toronto, Ontario Canada. Contact: Norm Looney, Chair, Executive Committee: e-mail: looneyn@cm.agr.ca

-17th world congress of soil science - "Confronting new realities in the 21st Century", 14-21 August.

Bankok, Thailand. Contact: Congress Office, Kasetsart University, Box 1048, Bankok 10903, Thailand.E-mail: o.sfst@nontrj.ku.ac.th

\section{SEPTEMBER 2002}

$\checkmark 50$ th annual meeting of the western international forest disease work conference, September (dates TBA).

Victoria, B.C. Canada. Contact: John Muir, B.C. Min. of Forests, Forest Practices Branch, 1450 Government Street, 1st Floor, Victoria, B.C.; Fax: 250-387-2136; e-mail: john.muir@ gems1.gov.bc.ca

\section{SEPTEMBER 2003}

- World forestry congress, 22-30 September.

Quebec City, Quebec Canada. Contact: Website: www.wfc2003.org/ 